\title{
Hypoxia-Inducible Factor-Proline Hydroxylase Inhibitor in the Treatment of Renal Anemia
}

\author{
Xiaofan Hu Jingyuan Xie Nan Chen \\ Department of Nephrology, Shanghai Ruijin Hospital, Shanghai Jiao Tong University School of Medicine, \\ Shanghai, China
}

\author{
Keywords \\ Chronic kidney disease · Anemia · Hypoxia-inducible \\ factor-proline hydroxylase inhibitor
}

\section{Abstract}

Background: Anemia is a common complication in CKD patients. Despite the use of iron and erythropoietin-stimulating agents, the control rate of anemia in CKD is not satisfying. Novel drugs are needed for anemia correction. Summary: HIF-PHI, hypoxia-inducible factor-proline hydroxylase inhibitor, a novel class of therapeutic agents, has been developed to treat anemia in CKD patients. Its main effects comprised boosting EPO production, enhancing iron utilization, and suppressing hepcidin production. Several stage 2 and stage 3 clinical trials have been run to test its efficacy and safety in both nondialysis and dialysis patients, of which the results are very encouraging. Here, we summarize the mechanism, clinical applications, and clinical trials of HIF-PHI in treating renal anemia in order to give an overview of the new drug in clinical practices. Key Messages: HIF-PHI is a novel therapeutic agent of treating renal anemia in CKD patients. It is quite effective in improving anemia, which is unaffected by inflammation. Besides, it may ameliorate lipid metabolism as well. Furthermore, the oral form may improve patients' compliances with treatment. Thus, it may be a good alternative of anemia correction in CKD patients.

(C) 2020 The Author(s)

Published by S. Karger AG, Basel

\section{Introduction}

Chronic kidney disease (CKD) has grown to be a worldwide public health problem, affecting $10.8 \%$ patients in China. Anemia is a common complication in CKD patients, accounting for $18.50 \sim 22.20 \%$ in nondialysis CKD patients $[1,2]$ and $30 \sim 80 \%$ (DOPPS4 and CNRDS 2011 data) in dialysis patients. Previous studies showed that anemia was associated with mortality, quality of life, cognition deficiency, renal function progression, and cardiovascular events [3-7]. Thus, the European Renal Best Practice position statement [8] recommends that hemoglobin should be maintained around $11 \sim 12 \mathrm{~g} / \mathrm{dL}$ in CKD patients. However, despite the use of iron and erythropoietin-stimulating agents (ESAs), the control rate of anemia in CKD patients is not satisfying, with only $63.1 \sim 90.7 \%$ meeting the goal, partly due to the karger@karger.com www.karger.com/kdd

Karger $\stackrel{\text { ' }}{5}$

GOPEN ACCESS
(C) 2020 The Author(s)

Published by S. Karger AG, Basel

This article is licensed under the Creative Commons AttributionNonCommercial-NoDerivatives 4.0 International License (CC BY NC-ND) (http://www.karger.com/Services/OpenAccessLicense) Usage and distribution for commercial purposes as well as any distribution of modified material requires written permission.
Nan Chen

Department of Nephrology, Shanghai Ruijin Hospital

Shanghai Jiao Tong University School of Medicine

No. 197 Ruijin Er Rd., Shanghai 200025 (China) cnrj100@126.com 
inflammation conditions in some patients [9]. Furthermore, the safety of ESAs remains a great concern in clinical practices. Several studies have shown that ESAs may provokehypertension, thrombosis, cardiovascular events, and even death in CKD patients [10-13]. One of the reasons for these side effects was that ESA can bring nonphysiological EPO level. Therefore, efforts should be taken in finding new drugs in treating renal anemia.

Anemia in CKD is mainly due to inadequate synthesis of erythropoietin, iron deficiency, inflammation, and reduced erythrocyte survival [14]. It is well recognized that HIF-2a plays an important role in regulating the expression of the EPO gene in renal EPO-producing cells [15, 16]. Thus, the inhibition of the enzymes in the HIF-2arelated pathway may boost EPO production, improving renal anemia. As a result, HIF-PHI, hypoxia-inducible factor-proline hydroxylase inhibitor, a novel class of therapeutic agents, has been developed to treat anemia in CKD patients. Several stage 2 and stage 3 clinical trials have been run to test its efficacy and safety in both nondialysis and dialysis patients, of which the results are very encouraging. Here, we summarize the mechanism, clinical applications, and clinical trials of HIF-PHI in treating renal anemia in order to give an overview of the new drug in clinical practices.

\section{Mechanism}

HIF, first discovered in the 1950s, is an important intracellular modulator of hypoxia-induced reactions [17, 18]. It comprises 2 different subunits, alfa and beta chains. Alfa chains have 3 main forms, which are all oxygen sensitive, whereas the beta chains are constitutively expressed $[19,20]$. Previous studies $[15,16,21,22]$ show that HIF2a plays an important role in regulating erythropoiesis through 3 different mechanisms: EPO production, iron absorption, and hepcidin suppression. Once hypoxia triggers the activation of HIF-2a, it facilitates the expression of EPO, increasing EPO production to stimulate erythropoiesis in bone marrow [23, 24]. Besides, HIF-2a also enhances the enteral iron absorption by boosting production of divalent metal transporter 1 and duodenal cytochrome $\mathrm{B}$, improving iron transportation from the intestinal lumen to the enterocytes $[25,26]$. Furthermore, it inhibits the production of hepcidin which suppresses the iron uptake and mobilization [21,27]. As a result, mature erythrocytes are produced for oxygen delivery to ameliorate the hypoxia in tissues. As soon as hypoxia is corrected, HIF-2a is quickly hydroxylated by prolyl hy- droxylase enzymes (PHD) and then degraded through uquibitination by proteasomes, stopping the downstream reactions [28]. PHDs have 3 isoforms with PHD2 acting more on HIF1 $\alpha$ and PHD3 more on HIF2 $\alpha$ [29].

In CKD patients, there is a discrepancy between oxygen supply and demand in the kidneys, disturbing the hypoxia-induced signaling in renal EPO-producing cells [30]. Inhibition of PH will restore the HIF-2a pathway and stimulate erythropoiesis in CKD patients, improving renal anemia. Thus, HIF-PHI has been developed and put into clinical trials to test its efficacy and safety in CKD patients.

\section{Clinical Trials}

The HIF-PHIs under investigation such as Roxadustat $^{\circledR}$, Daprodustat ${ }^{\circledR}$, Molidustat ${ }^{\circledR}$, and Vadadustat ${ }^{\circledR}$ are currently in phase 3 studies and all proved to be quite effective in treating renal anemia in CKD patients. The main differences of the 4 drugs comprise their various activities against 3 HIF-PHDs and the diverse influence on iron and lipid metabolism. Here is a summary of the performances of 4 drugs in clinical trials (Table 1).

\section{Roxadustat}

Roxadustat is the first HIF-PHI developed by Fibrogen almost a decade ago. It inhibited all 3 HIF-PHIs equally. The first clinical trial was started in 2005. With its great efficacy in renal anemia correction, it has been approved by the National Medical Products Administration for renal anemia in China in 2019. Besides, the phase 3 clinical trials of Roxadustat in the treatment of anemia in Chinese CKD patients have just been released. There were 2 parallel clinical trials focused on anemia treatment by Roxadustat in NDD or DD patients, and both the trials were led by Prof Chen Nan from Shanghai Ruijin Hospital.

In NDD patients, one phase II Chinese study [31] conducted from 2011 to 2012 enrolled 91 CKD patients with eGFR $10 \sim 60 \mathrm{~mL} / \mathrm{min} \times 1.73 \mathrm{~m}^{2}$ and randomized them into different Roxadustat starting doses or placebo. By the end of 8 weeks, the overall hemoglobin response rate (hemoglobin $\geq 1.0 \mathrm{~g} / \mathrm{dL}$ from baseline) was significantly higher in the Roxadustat group than in the placebo one (83.6 vs. $23.3 \%$ ). The mean maximum $\Delta \mathrm{Hb}$ was 1.82 and $2.59 \mathrm{~g} / \mathrm{dL}$ in the low- and high-dose cohorts, versus 0.65 $\mathrm{g} / \mathrm{dL}$ in the placebo group. Another phase II study [32] from 2013 to 2015 recruiting 107 Japanese NDD patients 
Table 1. Summary of 4 HIF-PHIs

\begin{tabular}{|c|c|c|c|c|c|c|c|c|}
\hline Drug name & $\begin{array}{l}\text { Activities against } 3 \text { HIF- } \\
\text { PHDs }\end{array}$ & $\begin{array}{l}\text { Ongoing } \\
\text { phase study }\end{array}$ & Dosages reported & $\begin{array}{l}\text { Frequencies } \\
\text { reported }\end{array}$ & $\begin{array}{l}\text { Comparison } \\
\text { with ESA in } \\
\text { DD }\end{array}$ & $\begin{array}{l}\text { Comparison } \\
\text { with ESA in } \\
\text { NDD }\end{array}$ & Iron metabolism & $\begin{array}{l}\text { Lipid } \\
\text { metabolism }\end{array}$ \\
\hline Roxadustat & PHD3 = PHD1 = PHD2 & Phase 3 & $\begin{array}{l}50-120 \mathrm{mg} \text { (based } \\
\text { on weight) }\end{array}$ & TIW, BIW & Noninferior & NR & $\begin{array}{l}\text { Both in DD and } \\
\text { NDD }\end{array}$ & $\begin{array}{l}\text { Both in DD } \\
\text { and NDD }\end{array}$ \\
\hline Daprodustat & PHD3 > PHD1 > PHD2 & Phase 3 & $5-100 \mathrm{mg}$ & Qd & Noninferior & NR & $\begin{array}{l}\text { Both in DD and } \\
\text { NDD }\end{array}$ & $\begin{array}{l}\text { In } \mathrm{DD} \text { and } \mathrm{NR} \\
\text { in NDD }\end{array}$ \\
\hline Molidustat & PHD3 = PHD1 = PHD2 & Phase 3 & $25 \sim 150 \mathrm{mg}$ & Qd & Noninferior & Noninferior & In NDD & Not obvious \\
\hline Vadadustat & PHD3 > PHD1 > PHD2 & Phase 3 & $150-600$ & Qd & NR & NR & $\begin{array}{l}\text { Both in DD and } \\
\text { NDD }\end{array}$ & NR \\
\hline
\end{tabular}

NR, not reported; HIF-PHIs, hypoxia-inducible factor-proline hydroxylase inhibitors; PHD, prolyl hydroxylase enzyme; ESA, erythropoietin-stimulating agent.

showed that patients treated with different regimens of Roxadustat had a mean increase in hemoglobin of $0.20 \pm$ $0.16 \sim 0.57 \pm 0.24 \mathrm{~g} / \mathrm{dL}$ by the end of 6 weeks, much higher than that of the placebo group $(-0.052 \pm 0.142 \mathrm{~g} / \mathrm{dL})$. Provenzano et al.'s [33] phase II study completed in 2012 showed a mean $\Delta \mathrm{Hb}$ varied from 0.6 to $1.7 \mathrm{~g} / \mathrm{dL}$ based on different Roxadustat regimens in the first 4 weeks. Patients with different levels of baseline CRP shared similar mean $\Delta \mathrm{Hb}$ values, indicating that Roxadustat-induced hemoglobin increases were independent of baseline CRP. All these studies confirmed Roxadustat's efficacy in improving renal anemia and demonstrated that Roxadustat augmented hemoglobin in a dose-dependent manner. Moreover, studies also showed that Roxadustat enhanced iron metabolism and reduced hepcidin in NDD patients. In Besarab et al.'s [34] phase II study conducted from 2008 to 2010 , compared with the placebo group, patients with Roxadustat had an evident rise in total iron-binding capacity (TIBC) $(41.8 \pm 45.4$ vs. $-7.6 \pm 26.6 \mu \mathrm{g} / \mathrm{dL})$, indicating an increased iron utilization. Besides, serum hepcidin level was significantly decreased in 1.5 and $2.0 \mathrm{mg} /$ $\mathrm{kg}$ Roxadustat dose groups by $-150 \pm 89.5$ and $-225 \pm 192$ $\mathrm{ng} / \mathrm{mL}$, respectively, whereas it was only $-17.8 \pm 114 \mathrm{ng} /$ $\mathrm{mL}$ in the placebo group. Iron deficiency and anemia were improved by more iron being absorbed from intestines and released from macrophages to participate in hemoglobin production due to TIBC improvement and serum hepcidin inhibition by Roxadustat. In addition to its effect on erythropoiesis, Roxadustat was reported to reduce cholesterol level as well. Provenzano et al. [33] reported a reduction in cholesterol by $26 \pm 30 \mathrm{mg} / \mathrm{dL}$ after 8 weeks of treatment. Considering the relatively smallsample and short-time follow-up of the previous studies,
Chen et al. [35] have carried out phase 3 clinical trials in 2015 2016 by enlarged cohorts and prolonged follow-up to better demonstrate the efficacy and long-term effect of Roxadustat. In total, 154 NDD CKD patients from 29 renal centers were randomized to receive Roxadustat or placebo for 8 weeks in a double-blind manner and then all switched to Roxadustat for an 18-week open-label period. During the randomization phase, the patients in the Roxadustat group had a mean increase of $1.9 \pm 1.2 \mathrm{~g} / \mathrm{dL}$ in hemoglobin, a mean reduction of $56.14 \pm 63.40 \mathrm{ng} / \mathrm{mL}$ in hepcidin, and a decrease of $40.6 \mathrm{mg} / \mathrm{dL}$ from baseline in cholesterol, while patients in the placebo group suffered a mean decrease of $0.4 \pm 0.8 \mathrm{~g} / \mathrm{dL}$ in hemoglobin, a mean reduction of $15.10 \pm 48.06 \mathrm{ng} / \mathrm{mL}$ in hepcidin, and a decrease of $7.7 \mathrm{mg} / \mathrm{dL}$ from baseline in cholesterol. During the open-label period, the hemoglobin was well maintained by Roxadustat. And, patients initially treated by placebo were witnessed to have an improvement in lipid metabolism as well (shown in Table 2).

In dialysis patients, Roxadustat has also been confirmed to improve anemia unaffected by inflammation, enhance iron utilization, and reduce cholesterol [31, 3639]. Whether it can replace ESAs to be the first-line therapy in dialysis patients has been explored in several clinical trials with no consensus reached. Chen et al. [39] enrolled $305 \mathrm{DD}$ patients with stable use of ESA and randomized them to either Roxadustat or epoetin alfa in a phase III study in 2015 2016. During the study period of 27 weeks, the 2 treatment shows no difference in anemia correcting $(\Delta \mathrm{Hb}$ : Roxadustat vs. epoetin alfa $0.7 \pm 1.1$ vs. $0.5 \pm 1.0 \mathrm{~g} / \mathrm{dL}, p>0.05$, hemoglobin response: 92.5 vs. $92.5 \%)$, partially aligned with Provenzano et al.'s [37] findings in a phase II study in 2010 2012. However, in the 
Table 2. Roxadustat's effects in NDD patients [32-35, 40]

\begin{tabular}{|c|c|c|c|c|c|c|c|}
\hline Study & No & Drugs & $\begin{array}{l}\text { Follow-up } \\
\text { time }\end{array}$ & Protocol & Primary endpoint & Response rate & Other benefits \\
\hline $\begin{array}{l}\text { Akizawa et al. } \\
\text { [32] (Japan, } \\
\text { mean eGFR: } \\
16.3 \mathrm{~mL} / \\
\mathrm{min} / 1.73 \mathrm{~m} \text { ) }\end{array}$ & 83 & $\begin{array}{l}\text { Roxadustat } \\
\text { versus } \\
\text { placebo }\end{array}$ & $24 \mathrm{wk}$ & $\begin{array}{l}\text { Placebo, } 50,70 \text {, and } 100 \mathrm{mg} \text { TIW for } \\
18 \text { weeks; if } \mathrm{Hb} 10 \sim 12 \mathrm{~g} / \mathrm{L} \text {, } \\
\text { Roxadustat switched to TIW or QW; } \\
\text { if not, continue TIW }\end{array}$ & $\begin{array}{l}\text { Rate of rise of } \mathrm{Hb} \text { within } \\
\text { the first } 6 \mathrm{wk}\end{array}$ & $\begin{array}{l}14.8 \text { versus } 81.5 \\
\text { versus } 100 \text { versus } \\
100 \%\end{array}$ & $\begin{array}{l}\text { 1. Hepcidin } \\
\text { reduction } \\
\text { 2. Increase in TIBC } \\
\text { 3. Reduction in } \\
\text { cholesterol }\end{array}$ \\
\hline $\begin{array}{l}\text { Besarab et al. } \\
\text { [34] (US, CKD } \\
\text { stage } 3-4 \text { ) }\end{array}$ & 116 & $\begin{array}{l}\text { Roxadustat } \\
\text { versus } \\
\text { placebo }\end{array}$ & $6 \mathrm{wk}$ & $\begin{array}{l}\text { Four dose cohorts } \\
\text { Roxadustat BIW }(0.7,1.0,1.5 \text {, and } \\
2.0 \mathrm{mg} / \mathrm{kg}) \text { versus placebo BIW } \\
\text { versus Roxadustat TIW }(0.7,1.0,1.5 \text {, } \\
\text { and } 2.0 \mathrm{mg} / \mathrm{kg}) \text { versus placebo TIW } \\
\text { for } 4 \text { weeks }\end{array}$ & $\begin{array}{l}\text { 1. Mean } \mathrm{Hb} \text { change } \\
(\Delta \mathrm{Hb}) \text { from baseline } \\
\text { 2. Proportion of } \mathrm{Hb} \\
\text { responders }(\Delta \mathrm{Hb} \geq 1.0 \\
\mathrm{g} / \mathrm{dL})\end{array}$ & $\begin{array}{l}\Delta \text { Hb: Roxadustat } \\
\text { group: } \\
0.8 \pm 0.9 \sim 2.2 \pm 0.8 \\
\text { g/dL, placebo: } \\
-0.1 \mathrm{~g} / \mathrm{dL} \\
\text { Response rate: } \\
\text { Roxadustat } \\
\text { group: } 30 \sim 100 \% \text {, } \\
\text { placebo: } 13 \%\end{array}$ & $\begin{array}{l}\text { 1. Hepcidin } \\
\text { reduction } \\
\text { 2. Increase in TIBC }\end{array}$ \\
\hline $\begin{array}{l}\text { Provenzano et } \\
\text { al. [33] (USA, } \\
\text { CKD stage } \\
3 \sim 4 \text { ) }\end{array}$ & 145 & Roxadustat & $16 \sim 24 \mathrm{wk}$ & $\begin{array}{l}\text { Randomization into } 6 \text { cohorts with } \\
\text { varying Roxadustat starting doses } \\
\text { and frequencies followed by } \\
\text { hemoglobin maintenance with } \\
\text { Roxadustat } 1-3 \text { times weekly } \\
\text { A: TIW, } 16 \mathrm{~W},(60,100 \text {, and } 140 \mathrm{mg} \\
\text { based on patient's weight) } \\
\text { B: TIW to BIW (maintenance), } \\
16 \mathrm{~W},(60,100 \text {, and } 140 \mathrm{mg} \text { based } \\
\text { on patient's weight) } \\
\text { C: TIW, } 24 \text { wk, } 50, \mathrm{mg} \\
\text { D: TIW, } 24 \mathrm{wk}, 100 \mathrm{mg} \\
\text { E: BIW to QW, } 24 \mathrm{wk},(70,100 \text {, and } \\
150 \mathrm{mg} \text { based on patient's weight) } \\
\text { F: TIW to BIW to QW, } 24 \mathrm{wk}, 70 \mathrm{mg}\end{array}$ & $\begin{array}{l}\text { The proportion of } \\
\text { patients achieving } \\
\text { hemoglobin increase of } \\
\geq 1.0 \mathrm{~g} / \mathrm{dL} \text { from baseline } \\
\text { and hemoglobi } n \text { of } \\
\geq 11.0 \mathrm{~g} / \mathrm{dL} \text { by } 17 \text { ( } 16 \\
\text { weeks of treatment) }\end{array}$ & $\begin{array}{l}\text { Overall: } 91.6 \% \\
\text { A, B, C, D, E, F: } \\
82.6,100,91.3 \\
95.8,95.8,84 \%\end{array}$ & $\begin{array}{l}\text { 1. Hepcin } \\
\text { reduction } \\
\text { 2. Not influenced } \\
\text { by inflammatory } \\
\text { status } \\
\text { 3. Cholesterol } \\
\text { reduction }\end{array}$ \\
\hline $\begin{array}{l}\text { Chen et al. }[40] \\
(\mathrm{eGFR}: 10 \sim 60 \\
\mathrm{mL} / \mathrm{min} / 1.73 \\
\left.\mathrm{~m}^{2}\right)\end{array}$ & 91 & $\begin{array}{l}\text { Roxadustat } \\
\text { versus } \\
\text { placebo }\end{array}$ & $\begin{array}{l}8 \text {-wk } \\
\text { treatment }\end{array}$ & $\begin{array}{l}\text { Randomization into } 3 \text { cohorts: } \\
\text { Placebo versus low dose }(1.1 \sim 1.75 \\
\mathrm{mg} / \mathrm{kg}, \text { TIW }) \text { versus high dose } \\
(1.5 \sim 2.25 \mathrm{mg} / \mathrm{kg}, \mathrm{TIW})\end{array}$ & $\begin{array}{l}\text { 1. The maximum } \mathrm{Hb} \\
\text { change from baseline } \\
\text { 2. The proportion of } \\
\text { subjects who were } \mathrm{Hb} \\
\text { responders (defined as } \\
\text { an Hb increase of } \geq 1.0 \\
\mathrm{~g} / \mathrm{dL} \text { from baseline at } \\
\text { any time) } \\
\text { 3. The proportion of } \\
\text { subjects who achieved } \\
\text { an Hb level of } \geq 11.0 \mathrm{~g} / \\
\mathrm{dL}\end{array}$ & $\begin{array}{l}\text { Placebo versus } \\
\text { low dose versus } \\
\text { high dose } \\
1.0 .65 \text { versus } \\
1.82 \text { versus } 2.59 \\
\text { g/dL } \\
2.25 .9 \text { versus } \\
88.5 \text { versus } 93.1 \% \\
3.6 .7 \text { versus } 50 \\
\text { versus } 71 \%\end{array}$ & $\begin{array}{l}\text { 1. Hepcin } \\
\text { reduction } \\
\text { 2. Increase in TIBC } \\
\text { 3. Improvement in } \\
\text { lipid metabolism }\end{array}$ \\
\hline Chen et al. [35] & 154 & $\begin{array}{l}\text { Roxadustat } \\
\text { versus } \\
\text { placebo }\end{array}$ & $\begin{array}{l}8 \text { wk } \\
\text { (Roxadustat } \\
\text { vs. placebo) } \\
+18 \text { wk } \\
\text { Roxadustat }\end{array}$ & $\begin{array}{l}\text { Randomization into } 3 \text { cohorts: } \\
\text { Placebo versus low dose }(70 \mathrm{mg} \text {, } \\
\text { TIW) versus high dose }(100 \mathrm{mg} \text {, } \\
\text { TIW) }\end{array}$ & $\begin{array}{l}\text { The mean change from } \\
\text { baseline in the } \\
\text { hemoglobin level during } \\
\text { weeks 7-9 }\end{array}$ & $\begin{array}{l}\text { Placebo versus } \\
\text { Roxadustat: } \\
0.4 \pm 0.8 \text { versus } \\
1.9 \pm 1.2 \mathrm{~g} / \mathrm{dL}\end{array}$ & $\begin{array}{l}\text { 1. Hepcin } \\
\text { reduction } \\
\text { 2. Increase in iron } \\
\text { utilization } \\
\text { 3. Improvement in } \\
\text { lipid metabolism }\end{array}$ \\
\hline
\end{tabular}

TIBC, total iron-binding capacity.

subgroups of patients with high-level CRP, Roxadustat resulted in a greater $\Delta \mathrm{Hb}$ of $0.9 \pm 1.0 \mathrm{~g} / \mathrm{dL}$ than epoetin alfa $(0.3 \pm 1.1 \mathrm{~g} / \mathrm{dL})$. In addition, compared with epoetin alfa, patients treated with Roxadustat had an increase in TIBC level (difference, $10.7 \pm 1.3 \mu \mathrm{mol} / \mathrm{L}$; 95\% CI: 8.1-13.3) and a decrease in hepcidin level (Roxadustat vs. epoetin alfa: 30.2 vs. $2.3 \mathrm{ng} / \mathrm{mL}$ ), indicating an improvement in iron utilization. Moreover, Roxadustat enhanced lipid metabolism, causing a greater decrease in total cholesterol than epoetin alfa (difference, $-22 \mathrm{mg}$ per deciliter; $95 \% \mathrm{CI}$ : -29 
Table 3. Roxadustat's effects in DD patients [37-40, 51]

\begin{tabular}{|c|c|c|c|c|c|c|c|}
\hline Study & No. & Drugs & $\begin{array}{l}\text { Follow- } \\
\text { up time }\end{array}$ & Protocol & Primary endpoint & Response rate & Other benefits \\
\hline $\begin{array}{l}\text { Akizawa et } \\
\text { al. [38] } \\
\text { (Japan) }\end{array}$ & $53 \mathrm{PD}$ & Roxadustat & $24 \mathrm{wk}$ & $\begin{array}{l}\text { ESA naïve }{ }^{\mathrm{a}} \text { patients: } 50 \text { or } \\
70 \mathrm{mg} \text { TIW } \\
\text { ESA-dependant patients: } \\
70 \text { or } 100 \mathrm{mg} \text { TIW }\end{array}$ & $\begin{array}{l}\text { At weeks } 18 \sim 24: \\
\text { Maintenance rate of } \mathrm{Hb} \\
\text { within } 10-12 \mathrm{~g} / \mathrm{dL} \\
\text { Average } \mathrm{Hb} \text { levels at } \\
\text { weeks } 18-24\end{array}$ & $\begin{array}{l}\text { At weeks } 18 \sim 24: \text { ESA } \\
\text { naïve: } 92.3 \%, \mathrm{Hb} \\
11.05 \pm 0.62 \mathrm{~g} / \mathrm{L} \\
\text { ESA dependant: } 74.4 \%, \mathrm{Hb} \\
10.93 \pm 0.61 \mathrm{~g} / \mathrm{L}\end{array}$ & Reduction of hepcidin \\
\hline $\begin{array}{l}\text { Besarab et } \\
\text { al. [51] }\end{array}$ & $\begin{array}{l}48 \mathrm{HD}+ \\
12 \mathrm{PD}\end{array}$ & Roxadustat & $12 \mathrm{wk}$ & $\begin{array}{l}\text { Roxadustat (TIW initial } \\
\text { dose: } 1.0-1.7 \mathrm{mg} / \mathrm{kg} \text { per } \\
\text { dose, adjustment every } 4 \\
\text { weeks based on } \mathrm{Hb} \text { ) to } 4 \\
\text { groups: HD with oral } \\
\text { iron, HD with IV iron, PD } \\
\text { with IV iron and HD } \\
\text { without iron }\end{array}$ & $\begin{array}{l}\text { The maximum } \mathrm{Hb} \\
\text { change from baseline } \\
\text { during the last } 10 \text { weeks } \\
\text { of the dosing period }\end{array}$ & $\begin{array}{l}\Delta \text { Hbmax: HD with oral } \\
\text { iron: } 3.5 \pm 0.2 \text {; HD with IV } \\
\text { iron: } 3.5 \pm 0.4 \text {; PD with oral } \\
\text { iron: } 3.5 \pm 0.2 \text {; HD without } \\
\text { iron: } 2.8 \pm 0.2 \\
\Delta \text { hepcidin: HD without } \\
\text { iron: }-63.4 \pm 13.3 \text {; HD + PD } \\
\text { with oral iron: }-48.2 \pm 18.2 \text {; } \\
\text { HD with IV iron: } \\
-12.6 \pm 31.6\end{array}$ & $\begin{array}{l}\text { 1. Hepcidin reduction } \\
\text { 2. TIBC increase } \\
\text { 3. Not influenced by } \\
\text { inflammatory status }\end{array}$ \\
\hline $\begin{array}{l}\text { Provenzano } \\
\text { et al. [37] }\end{array}$ & $\begin{array}{l}\text { Part 1: } \\
42\end{array}$ & $\begin{array}{l}\text { Roxadustat } \\
\text { versus } \\
\text { epoetin alfa }\end{array}$ & $\begin{array}{l}\text { Part 1: } \\
6 \mathrm{wk}\end{array}$ & $\begin{array}{l}\text { Roxadustat }(1.0,1.5,1.8 \text {, } \\
\text { or } 2.0 \mathrm{mg} / \mathrm{kg}) \mathrm{TIW} \text { versus } \\
\text { epoetin alfa, IV, TIW }\end{array}$ & $\begin{array}{l}\text { Hb level response (end- } \\
\text { of-treatment } \mathrm{Hb} \text { level } \\
\text { change of }-0.5 \mathrm{~g} / \mathrm{dL} \text { or } \\
\text { greater from baseline) }\end{array}$ & $\begin{array}{l}\text { Roxadustat versus epoetin } \\
\text { alfa: } 79 \text { versus } 33 \%\end{array}$ & $\begin{array}{l}\text { 1. Hepcidin reduction } \\
\text { 2. Cholesterol } \\
\text { reduction } \\
\text { 3. Not influenced by } \\
\text { inflammation status }\end{array}$ \\
\hline $\begin{array}{l}\text { Provenzano } \\
\text { et al. [37] }\end{array}$ & $\begin{array}{l}\text { Part 2: } \\
83\end{array}$ & $\begin{array}{l}\text { Roxadustat } \\
\text { versus } \\
\text { epoetin alfa }\end{array}$ & $\begin{array}{l}\text { Part 2: } \\
19 \text { wk }\end{array}$ & $\begin{array}{l}\text { Roxadustat (from } \\
\text { different cohorts) versus } \\
\text { epoetin alfa, IV, TIW }\end{array}$ & $\begin{array}{l}\text { Hb level response (mean } \\
\text { Hb level } \geq 11.0 \mathrm{~g} / \mathrm{dL} \\
\text { during the last } 4 \\
\text { treatment weeks) }\end{array}$ & $\begin{array}{l}\text { Roxadustat versus epoetin } \\
\text { alfa: } 51 \text { versus } 36 \%\end{array}$ & $\begin{array}{l}\text { 1. Hepcidin reduction } \\
\text { 2. Cholesterol } \\
\text { reduction } \\
\text { 3. Not influenced by } \\
\text { inflammation status }\end{array}$ \\
\hline $\begin{array}{l}\text { Chen et al. } \\
{[40]}\end{array}$ & 87 & $\begin{array}{l}\text { Roxadustat } \\
\text { versus } \\
\text { epoetin alfa }\end{array}$ & $6 \mathrm{wk}$ & $\begin{array}{l}\text { Roxadustat }(1.1 \sim 1.8, \\
1.5 \sim 2.3 \text {, and } 1.7 \sim 2.3 \mathrm{mg} / \\
\mathrm{kg}) \text { TIW versus epoetin } \\
\text { alfa }\end{array}$ & $\begin{array}{l}\text { Successful dose } \\
\text { conversion\# percentage }\end{array}$ & $\begin{array}{l}\text { Roxadustat }(1.1 \sim 1.8, \\
1.5 \sim 2.3 \text {, and } 1.7 \sim 2.3 \mathrm{mg} / \\
\mathrm{kg}) \text { TIW versus epoetin } \\
\text { alfa: } 59.1 \text { versus } 88.9 \text { versus } \\
100 \text { versus } 50 \%\end{array}$ & $\begin{array}{l}\text { 1. Hepcidin reduction } \\
\text { 2. Increase in TIBC } \\
\text { 3. Cholesterol } \\
\text { reduction }\end{array}$ \\
\hline $\begin{array}{l}\text { Chen et al. } \\
\text { [39] }\end{array}$ & 305 & $\begin{array}{l}\text { Roxadustat } \\
\text { versus } \\
\text { epoetin alfa }\end{array}$ & $26 \mathrm{wk}$ & $\begin{array}{l}\text { Roxadustat (100, } 120 \mathrm{mg}) \\
\text { TIW versus epoetin alfa }\end{array}$ & $\begin{array}{l}\text { Mean change in } \\
\text { hemoglobin level from } \\
\text { baseline to the average } \\
\text { level during week } 23 \\
\text { through } 27\end{array}$ & $\begin{array}{l}\text { Roxadustat versus epoetin } \\
\text { alfa: } 0.7 \pm 1.1 \text { versus } 0.5 \pm 1.0 \\
\text { g/dL }\end{array}$ & $\begin{array}{l}\text { 1. Hepcidin reduction } \\
\text { 2. TIBC improvement } \\
\text { 3. Cholesterol } \\
\text { reduction } \\
\text { 4. Not influenced by } \\
\text { inflammatory status }\end{array}$ \\
\hline
\end{tabular}

TIBC, total iron-binding capacity; ESA, erythropoietin-stimulating agent. ${ }^{\text {a }}$ Patients not receving ESA before.

to -16). The study concluded that oral Roxadustat was noninferior to parenteral epoetin alfa in patients undergoing dialysis. In another phase II Chinese study in 2011 2012, 87 DD patients with stable doses of ESA were randomized to either different regimens of Roxadustat or continuation of epoetin alfa. After 6 weeks, 59.1, 88.9, and $100 \%$ of the low-, medium- and high-dose Roxadustattreated subjects maintained their hemoglobin levels versus only $50 \%$ of the epoetin alfa ones, which concluded a superiority of Roxadustat over epoetin alfa [40]. Although the results varied among different studies, Roxadustat was shown to be at least no inferior to epoetin alfa and could be a good alternative of ESA in anemia correction in DD patients (shown in Table 3).

With regard to the safety issues, Roxadustat has demonstrated its safety with only $0 \sim 14.2 \%$ drug-related serious adverse events recorded, which mainly comprised vascular access complications, infections, and cardiac disorders. The main adverse events reported are diarrhea, nausea, nasopharygitis, hyperkalemia, and hypertension (shown in Table 4). In general, Roxadustat is quite effective in renal anemia correction unaffected by inflammation and could be a good alternative of anemia correction in CKD patients. 
Table 4. Side effects of Roxadustat [32-35, 37-40, 51]

\begin{tabular}{|c|c|c|c|c|}
\hline Study & $\begin{array}{l}\text { Patients using } \\
\text { Roxadustat }\end{array}$ & $\begin{array}{l}\mathrm{AE} \\
\%\end{array}$ & $\begin{array}{l}\text { SAE, } \\
\%\end{array}$ & Frequent adverse events \\
\hline $\begin{array}{l}\text { Tadao et al. [32] } \\
\text { (Japan) }\end{array}$ & 80 & 78.8 & 13.8 & $\begin{array}{l}\text { Gastrointestinal disorders: diarrhea }(27.5 \%) \text { and nausea }(10 \%) \\
\text { Infections: nasopharyngitis }(26.3 \%) \\
\text { Declining kidney function }(6.3 \%) \\
\text { Musculoskeletal and connective tissue disorders: muscle spasm }(1.3 \%) \\
\text { Respiratory, thoracic, and mediastinal disorders: cough }(1.3 \%)\end{array}$ \\
\hline $\begin{array}{l}\text { Tadao et al. [38] } \\
\text { (Japan) }\end{array}$ & 56 & 87.5 & 14.3 & $\begin{array}{l}\text { Gastrointestinal disorders: diarrhea }(7.1 \%) \text {, vomiting }(7.1 \%) \\
\text { Infections: nasopharyngitis }(25 \%) \text {, catheter site infection }(7.1 \%) \\
\text { Musculoskeletal and connective tissue disorders: back pain }(8.9 \%) \\
\text { Skin and subcutaneous tissue disorders: pruritis }(5.4 \%)\end{array}$ \\
\hline Besarab et al. [51] & 60 & 50 & 10 & Hypertension ( $10 \%$, the most frequent), all the other AE were $\leq 5 \%$ \\
\hline Besarab et al. [34] & 88 & 59.1 & 5 & $\begin{array}{l}\text { Gastrointestinal disorders: diarrhea }(9.1 \%) \\
\text { Neurologic disorders: headache }(6.8 \%) \text {, dizziness }(2.3 \%) \text {, insomnia }(2.3 \%) \\
\text { Electrolytes disorder: hyperkalemia }(4.5 \%) \\
\text { Others: fatigue }(4.5 \%) \text {, back pain }(4.5 \%) \text {, peripheral edema }(3.4 \%) \text {, seasonal allergy }(1.1 \%) \text {, UTI }(1.1 \%)\end{array}$ \\
\hline $\begin{array}{l}\text { Provenzano et al. } \\
{[37]}\end{array}$ & 106 & 63.9 & 24.1 & $\begin{array}{l}\text { Frequent SAE } \\
\text { Gastrointestinal disorders: Gastrointestinal hemorrhage }(0.9 \%) \\
\text { Cardiac disorders: cardiac failure }(1.9 \%) \text {, ACS }(1.9 \%) \\
\text { Infections: gastroenteritis (1.9\%) } \\
\text { Metabolism and nutrition disorders: hyperkalemia }(0.9 \%) \\
\text { Neurologic disorders: stroke }(1.9 \%) \\
\text { Vascular disorders: AV fistula thrombosis }(0.9 \%) \\
\text { Hypersensitivity }(0.9 \%)\end{array}$ \\
\hline $\begin{array}{l}\text { Provenzano et al. } \\
{[33]}\end{array}$ & 145 & 80 & 24.1 & $\begin{array}{l}\text { Gastrointestinal disorders: nausea }(9.7 \%) \text {, diarrhea }(8.3 \%) \\
\text { Cardiac disorders: cardiac failure }(3.4 \%) \\
\text { Infections: sinusitis }(5.5 \%) \text {, UTI }(9.7 \%) \text {, nasopharingitis }(9 \%) \\
\text { Metabolism and nutrition disorders: hyponatremia }(2.1 \%) \\
\text { Neurologic disorders: dizziness }(6.2 \%) \text {, headache }(5.5 \%) \\
\text { Skin and subcutaneous tissue disorders }(0.9 \%) \\
\text { Vascular disorders: hypertension }(7.6 \%) \text {, peripheral edema }(12.4 \%) \\
\text { Renal disorders: renal failure }(4.9 \%)\end{array}$ \\
\hline Chen et al. [40] & 135 & 50 & 5.93 & $\begin{array}{l}\text { Gastrointestinal disorders: decreased appetite }(4 \%) \text {, nausea }(3 \%) \text {, diarrhea }(2 \%) \\
\text { Infections: nasopharingitis (1\%), upper respiratory tract infection }(4 \%) \\
\text { Metabolism and nutrition disorders: hyperkalemia (4\%) } \\
\text { Neurologic disorders: dizziness }(3 \%) \\
\text { Vascular disorders: hypertension }(5 \%) \\
\text { Renal disorders: renal failure }(3 \%)\end{array}$ \\
\hline Chen et al. [39] & 204 & 47.1 & 14.2 & $\begin{array}{l}\text { Gastrointestinal disorders: vomiting }(5.9 \%) \text {, liver function perturbation }(5.4 \%) \\
\text { Infections: upper respiratory tract infection }(18.1 \%) \\
\text { Cardioascular disorders: hypertension }(12.3 \%) \text {, chest discomfort }(6.4 \%) \text {, hypotension }(4.9 \%) \\
\text { Metabolism disorder: hyperkalemia }(7.4 \%) \\
\text { Neurologic disorders: dizziness }(4.9 \%)\end{array}$ \\
\hline Chen et al. [35] & 101 & 37 & 9 & $\begin{array}{l}\text { Gastrointestinal disorders: gastrointestinal hemorrhage }(1 \%) \\
\text { Infections: upper respiratory tract infection }(5 \%) \text {, pyrexia }(2 \%) \\
\text { Metabolism and nutrition disorders: hyperkalemia (16\%), metabolic acidosis }(12 \%) \text {, gout }(1 \%) \\
\text { Neurologic disorders: dizziness (1\%) cardiovascular disorders: hypertension }(6 \%) \text {, peripheral edema }(7 \%) \\
\text { Renal disorders: ESRD (1\%), chronic glomerulonephritis }(1 \%)\end{array}$ \\
\hline
\end{tabular}

\section{Daprodustat}

Daprodustat was developed in 2007 by GSK. It inhibits all 3 PHDs with a preference for PHD1 and PHD3. The clinical trials on renal anemia correction were started in
2008 and showed that Daprodustat was also effective in improving anemia and enhancing iron utilization in CKD patients, whereas its effects on lipid metabolism was only reported in DD patients [41-45]. Holdstock et al. [42] enrolled 156 CKD patients in a phase II study in 2013 2014, 
including $73 \mathrm{NDD}$ and $83 \mathrm{DD}$, and randomized them to either Daprodustat or control (placebo for NDD, EPO for $\mathrm{HD}$ ). By the end of 4 weeks, the observed $\Delta \mathrm{Hb}$ in different Daprodustat dose groups ranged from $-0.12 \pm 0.51$ to $0.95 \pm 0.66 \mathrm{~g} / \mathrm{dL}$ in NDD and $-1.06 \pm 0.83$ to $-0.08 \pm 0.63$ $\mathrm{g} / \mathrm{dL}$ in $\mathrm{DD}$, versus $-0.23 \pm 0.51 \mathrm{~g} / \mathrm{dL}$ in placebo and -0.25 $\pm 0.81 \mathrm{~g} / \mathrm{dL}$ in EPO. Besides, the study also showed a decrease in hepcidin and an increase in TIBC in patients with Daprodustat. In Akizawa et al.'s [45] phase II study in 2012 2013 with 86 DD patients enrolled, a mean hemoglobin change of $-0.28 \sim 0.97 \mathrm{~g} / \mathrm{dL}$ was witnessed in Daprodustat groups as compared to $-1.41 \mathrm{~g} / \mathrm{dL}$ in placebo, aligning with previous findings [41-44]. Moreover, the study also demonstrated a modest decrease in lipid parameters in DD patients treated with Daprodustat. The main serious drug-related adverse events reported varied from 1.3 to $18 \%$, which mainly comprised cardiac disorders [41-45]. Given that the published studies on Daprodustat are relatively small and short, prolonged and enlarged clinical trials are needed for further assessment of the long-term effects and safety issues of Daprodustat in CKD patients.

\section{Molidustat}

Molidudstat was a predominantly HIF-2a-stabilizing prolyl hydroxylase inhibitor developed by Akebia. Like Roxadustat and Daprodustat, Molidustat was shown by the Dialogue studies to be effective in treating renal anemia. The Dialogue studies consisted in 3 separate trials: Dialogue 1 phase II study conducted in 2014 2015 was to compare Molidustat with placebo in NDD patients not receiving ESA, while Dialogue 2 and 4 phase II study carried out in 2013 2015 centered on comparison between ESA and Molidustat in NDD and DD patients, respectively. In Dialogue 1, Molidustat was revealed to be associated with a mean increase in hemoglobin levels by 1.4$2.0 \mathrm{~g} / \mathrm{dL}$ after 16 weeks of administration. Dialogue 2 and 4 illustrated that compared with EPO, Molidustat maintained hemoglobin level with an estimated difference of up to $0.6 \mathrm{~g} / \mathrm{dL}$ in NDD and -0.1 to $0.4 \mathrm{~g} / \mathrm{dL}$ in DD patients [46]. To further observe its long-term effect, the Dialogue studies were extended to 52 weeks with NDD patients initially treated with placebo all switching to Molidustat. By the end of the study, hemoglobin was well maintained in both ESA and Molidustat groups with similar proportions of adverse events. Thus, the study concluded that Molidustat could be a good alternative of ESA in CKD patients. Besides, in NDD patients, a decrease in hepcidin and an augmentation of TIBC were observed in the Molidustat group, implicating an enhanced iron utilization while no similar changes were witnessed in DD patients [47].

In brief, Molidustat was effective in treating renal anemia and may be a good alternative of ESA in CKD patients. Yet, unlike Roxadustat, its effects on iron utilization were only observed in NDD patients. More clinical trials with more DD patients enrolled should be done to further explore the issues.

\section{Vadadustat}

Vadadustat was developed by Bayer Healthcare in 2008. Similar to the 3 drugs mentioned above, published phase II studies [48-50] also showed that Vadadustat improved renal anemia and enhanced iron utilization in a dose-dependent manner in CKD patients. However, the clinical trials were relatively small with a comparatively short follow-up time. Besides, in DD patients, only comparisons of different regimens of Vadadustat were performed with no control group included. Therefore, more studies should be done in the field to confirm Vadadustat's effects.

Among the 4 HIF-PHIs, Roxadustat was the first HIFPHIs tested in clinical studies to prove its efficacy of renal anemia correction in both NDD and DD patients. In addition, it could also improve lipid metabolism and iron utilization in all CKD patients. Besides, the lower frequency of Roxadustat administration may enhance patients' compliance. Daprodustat has a similar effect as Roxadustat while its lipid metabolism improvement was only reported in DD patients. The other 2 HIF-PHIs are more recently developed with less data which should await more clinical trials for further assessment. All 4 HIF-PHIs were well tolerated in published studies with low rate of serious adverse events.

\section{Conclusion}

HIF-PHI is a novel class of therapeutic agents in treating renal anemia. Its main effects comprised boosting EPO production, enhancing iron utilization, and suppressing hepcidin production. Compared with ESA, it is unaffected by inflammation and may ameliorate lipid metabolism. Besides, the oral form may improve patients' compliance with treatment. Viewing its success in CKD patients, ongoing clinical trials have been ex- 
tended to treat MDS and chemotherapy-induced anemia. As the drug is newly developed, prolonged and enlarged clinical trials are needed for further assessment of the long-term effects and safety issues of HIF-PHI in CKD patients before its widely application in the market.

\section{Conflict of Interest Statement}

The authors have no conflicts of interest to disclose.

\section{Funding Sources}

This work was supported by grants from the National Key Research and Development Program of China (2016YFC0904100) and the National Natural Science Foundation of China (Nos. 81870460, 81570598, and 81370015).

\section{Author Contributions}

C.N. designed the research; H.X.F. contributed to reference collection and drafting the manuscript; X.J.Y. and C.N. provided substantial guidance and revised the manuscript. All authors read and approved the final manuscript.

\section{References}

1 Dmitrieva O, de Lusignan S, Macdougall IC, Gallagher H, Tomson C, Harris K, et al. Association of anaemia in primary care patients with chronic kidney disease: cross sectional study of quality improvement in chronic kidney disease (QICKD) trial data. BMC Nephrol. 2013 Jan 25;14:24.

2 Stauffer ME, Fan T. Prevalence of anemia in chronic kidney disease in the United States. PLoS One. 2014;9(1):e84943.

3 Levin A, Djurdjev O, Duncan J, Rosenbaum D, Werb R. Haemoglobin at time of referral prior to dialysis predicts survival: an association of haemoglobin with long-term outcomes. Nephrol Dial Transplant. 2006 Feb; 21(2):370-7.

4 Walker AM, Schneider G, Yeaw J, Nordstrom B, Robbins S, Pettitt D. Anemia as a predictor of cardiovascular events in patients with elevated serum creatinine. J Am Soc Nephrol. 2006;17(8):2293-8.

5 Singh NP, Sahni V, Wadhwa A, Garg S, Bajaj SK, Kohli R, et al. Effect of improvement in anemia on electroneurophysiological markers (P300) of cognitive dysfunction in chronic kidney disease. Hemodial Int. 2010;10(3): $267-73$.

6 Xie J, Kiryluk K, Wang W, Wang Z, Guo S, Shen $\mathrm{P}$, et al. Predicting progression of IgA nephropathy: new clinical progression risk score. PLoS One. 2012;7(6):e38904.

7 Eriksson D, Goldsmith D, Teitsson S, Jackson J, van Nooten F. Cross-sectional survey in CKD patients across Europe describing the association between quality of life and anaemia. BMC Nephrol. 2016;17(1):97.

8 Francesco L, Peter B, Adrian C, Angel DF, Lucia DV, David G, et al. Kidney disease: improving global outcomes guidelines on anaemia management in chronic kidney disease: a European Renal Best Practice position statement. Nephrol Dial Transplant. 2013;28(6): 1346-59.

9 Zuo L, Wang M, Hou F, Yan Y, Chen N, Qian J, et al. Anemia management in the China dialysis outcomes and practice patterns study. Blood Purif. 2016;42(1):33-43.
10 Singh AK, Szczech L, Tang KL, Barnhart H, Sapp S, Wolfson M, et al. Correction of anemia with epoetin alfa in chronic kidney disease. N Engl J Med. 2006;355(20):2085-98.

11 Pfeffer MA, Burdmann EA, Chen CY, Cooper ME, de Zeeuw D, Eckardt KU, et al. A trial of darbepoetin alfa in type 2 diabetes and chronic kidney disease. N Engl J Med. 2009;361(21): 2019-32.

12 Drüeke TB, Francesco L, Naomi C, Kai-Uwe E, Macdougall IC, Dimitrios T, et al. Anemia treatment in patients with chronic kidney disease. N Engl J Med. 2013;368(4):387-9.

13 Ioannis K, Mansour A, Trikalinos TA, Balk EM, Jaber BL. Dose of erythropoiesis-stimulating agents and adverse outcomes in CKD: a metaregression analysis. Am J Kidney Dis. 2013;61(1):44-56.

14 Babitt JL, Lin HY. Mechanisms of anemia in CKD. J Am Soc Nephrol. 2012 Oct;23(10): 1631-4.

15 Christina W, Zaneta Z, Jens K, Erdmann VA, Ulrich F, Michael W, et al. Differentiating the functional role of hypoxia-inducible factor (HIF)-1alpha and HIF-2alpha (EPAS-1) by the use of RNA interference: erythropoietin is a HIF-2alpha target gene in Hep3B and Kelly cells. FASEB J. 2004;18(12):1462-4.

16 Percy MJ, Furlow PW, Lucas GS, Li X, Lappin TR, McMullin MF, et al. A gain-of-function mutation in the HIF2A gene in familial erythrocytosis. N Engl J Med. 2008;358(2):162.

17 Windsor JS, Rodway GW. Heights and haematology: the story of haemoglobin at altitude. Postgrad Med J. 2007;83(977):148-51.

18 Locatelli F, Fishbane S, Block GA, Macdougall IC. Targeting hypoxia-inducible factors for the treatment of anemia in chronic kidney disease patients. Am J Nephrol. 2017;45(3): 187-99.

19 Semenza GL. Regulation of mammalian O2 homeostasis by hypoxia-inducible factor 1 . Annu Rev Cell Dev Biol. 2016;15(15):551-78.

20 Haase VH. HIF-prolyl hydroxylases as therapeutic targets in erythropoiesis and iron metabolism. Hemodial Int. 2017;21(Suppl 1): S110.
21 Ga LN, Caroline C, Lydie V, Jean Louis D, Xavier B, Isabelle D, et al. The gene encoding the iron regulatory peptide hepcidin is regulated by anemia, hypoxia, and inflammation. J Clin Invest. 2002;110(7):1037-44.

22 Haase VH. Regulation of erythropoiesis by hypoxia-inducible factors. Blood Rev. 2013 Jan;27(1):41-53.

23 Marzia S, Kan D, Quiyang Z, Yavuz O, Bennett MJ, Michael B, et al. HIF-2alpha regulates murine hematopoietic development in an erythropoietin-dependent manner. Blood. 2005;105(8):3133.

24 Percy MJ, Furlow PW, Lucas GS, Li X, Lappin TR, McMullin MF, et al. A gain-of-function mutation in the HIF2A gene in familial erythrocytosis. N Engl J Med. 2008 Jan 10;358(2): 162-8.

25 Mastrogiannaki M, Matak P, Keith B, Simon MC, Vaulont S, Peyssonnaux C. HIF-2alpha, but not HIF-1alpha, promotes iron absorption in mice. J Clin Invest. 2009;119(5):115966.

26 Maria M, Pavle M, Stéphanie D, Jean-Christophe D, Sophie V, Carole PJB. Deletion of HIF-2 $\alpha$ in the enterocytes decreases the severity of tissue iron loading in hepcidin knockout mice. Blood. 2012;119(2):587.

27 Maria M, Pavle M, Mathieu JRR, Stéphanie D, Patrick M, Sophie V, et al. Hepatic hypoxiainducible factor-2 down-regulates hepcidin expression in mice through an erythropoietin-mediated increase in erythropoiesis. Haematologica. 2012;97(6):827-34.

28 Warnecke C, Griethe W, Weidemann A, Jürgensen JS, Willam C, Bachmann S, et al. Activation of the hypoxia-inducible factor-pathway and stimulation of angiogenesis by application of prolyl hydroxylase inhibitors. FASEB J. 2003;17(9):1186.

29 Appelhoff RJ, Tian YM, Raval RR, Turley H, Harris AL, Pugh CW, et al. Differential function of the prolyl hydroxylases PHD1, PHD2, and PHD3 in the regulation of hypoxia-inducible factor. J Biol Chem. 2004 Sep 10; 279(37):38458-65. 
30 Locatelli F, Fishbane S, Block GA, Macdougall IC. Targeting hypoxia-inducible factors for the treatment of anemia in chronic kidney disease patients. Am J Nephrol. 2017;45(3): 187.

31 Chen N, Qian J, Chen J, Yu X, Mei C, Hao C, et al. Phase 2 studies of oral hypoxia-inducible factor prolyl hydroxylase inhibitor FG-4592 for treatment of anemia in China. Nephrol Dial Transplant. 2017;32(8):1373.

32 Akizawa T, Iwasaki M, Otsuka T, Reusch M, Misumi T. Roxadustat treatment of chronic kidney disease-associated anemia in Japanese patients not on dialysis: a phase 2, randomized, double-blind, placebo-controlled trial. Adv Ther. 2019;36(6):1438-54.

33 Provenzano R, Besarab A, Sun CH, Diamond SA, Durham JH, Cangiano JL, et al. Oral hypoxia-inducible factor prolyl hydroxylase inhibitor roxadustat (FG-4592) for the treatment of anemia in patients with CKD. Clin J Am Soc Nephrol. 2016 Jun 6;11(6):982-91.

34 Besarab A, Provenzano R, Hertel J, Zabaneh R, Klaus SJ, Lee T, et al. Randomized placebocontrolled dose-ranging and pharmacodynamics study of roxadustat (FG-4592) to treat anemia in nondialysis-dependent chronic kidney disease (NDD-CKD) patients. Nephrol Dial Transplant. 2015 Oct;30(10): 1665-73.

35 Chen N, Hao C, Peng X, Lin H, Yin A, Hao L, et al. Roxadustat for anemia in patients with kidney disease not receiving dialysis. $\mathrm{N}$ Engl J Med. 2019 Sep;381(11):1001-10.

36 Besarab A, Chernyavskaya E, Motylev I, Shutov E, Kumbar LM, Gurevich K, et al. Roxadustat (FG-4592): correction of anemia in incident dialysis patients. J Am Soc Nephrol. 2015;27(4):1225.
37 Provenzano R, Besarab A, Wright S, Dua S, Zeig S, Nguyen P, et al. Roxadustat (FG-4592) versus epoetin alfa for anemia in patients receiving maintenance hemodialysis: a phase 2 , randomized, 6- to 19-week, open-label, active-comparator, dose-ranging, safety and exploratory efficacy study. Am J Kidney Dis. 2016 Jun;67(6):912-24.

38 Akizawa T, Otsuka T, Reusch M, Ueno M. Intermittent oral dosing of roxadustat in peritoneal dialysis chronic kidney disease patients with anemia: a randomized, phase 3, multicenter, open冈label study. Ther Apher Dial. 2019;24(2):115-25.

39 Chen N, Hao C, Liu B-C, Lin H, Wang C, Xing $\mathrm{C}$, et al. Roxadustat treatment for anemia in patients undergoing long-term dialysis. $\mathrm{N}$ Engl J Med. 2019;381(11):1011-22.

40 Chen N, Qian J, Chen J, Yu X, Mei C, Hao C, et al. Phase 2 studies of oral hypoxia-inducible factor prolyl hydroxylase inhibitor FG-4592 for treatment of anemia in China. Nephrol Dial Transplant. 2017;32(8):1373-86.

41 Brigandi RA, Johnson B, Oei C, Westerman M, Olbina G, de Zoysa J, et al. A novel hypoxia-inducible factor-prolyl hydroxylase inhibitor (GSK1278863) for anemia in CKD: a 28day, phase $2 \mathrm{~A}$ randomized trial. Am J Kidney Dis. 2016 Jun;67(6):861-71.

42 Holdstock L, Meadowcroft AM, Maier R, Johnson BM, Jones D, Rastogi A, et al. Fourweek studies of oral hypoxia-inducible factorprolyl hydroxylase inhibitor GSK1278863 for treatment of anemia. J Am Soc Nephrol. 2016 Apr;27(4):1234-44.

43 Cizman B, Sykes AP, Paul G, Zeig S, Cobitz AR. An exploratory study of daprodustat in erythropoietin-hyporesponsive subjects. Kidney Int Rep. 2018 Jul;3(4):841-50.

44 Meadowcroft AM, Cizman B, Holdstock L, Biswas N, Johnson BM, Jones D, et al. Daprodustat for anemia: a 24-week, open-label, randomized controlled trial in participants on hemodialysis. Clin Kidney J. 2019 Feb;12(1): $139-48$.
45 Akizawa T, Tsubakihara Y, Nangaku M, Endo Y, Nakajima H, Kohno T, et al. Effects of daprodustat, a novel hypoxia-inducible factor prolyl hydroxylase inhibitor on anemia management in Japanese hemodialysis subjects. Am J Nephrol. 2017;45(2):127-35.

46 Macdougall IC, Akizawa T, Berns JS, Bernhardt T, Krueger T. Effects of Molidustat in the treatment of anemia in CKD. Clin J Am Soc Nephrol. 2019 Jan 7;14(1):28-39.

47 Akizawa T, Macdougall IC, Berns JS, Yamamoto $\mathrm{H}$, Taguchi M, Iekushi K, et al. Iron regulation by Molidustat, a daily oral hypoxiainducible factor prolyl hydroxylase inhibitor, in patients with chronic kidney disease. Nephron. 2019;143(4):243-54.

48 Pergola PE, Spinowitz BS, Hartman CS, Maroni $\mathrm{BJ}$, Haase $\mathrm{VH}$. Vadadustat, a novel oral HIF stabilizer, provides effective anemia treatment in nondialysis-dependent chronic kidney disease. Kidney Int. 2016 Nov;90(5): 1115-22.

49 Martin ER, Smith MT, Maroni BJ, Zuraw QC, deGoma EM. Clinical trial of Vadadustat in patients with anemia secondary to stage 3 or 4 chronic kidney disease. Am J Nephrol. 2017; 45(5):380-8.

50 Haase VH, Chertow GM, Block GA, Pergola PE, deGoma EM, Khawaja Z, et al. Effects of Vadadustat on hemoglobin concentrations in patients receiving hemodialysis previously treated with erythropoiesis-stimulating agents. Nephrol Dial Transplant. 2019 Jan 1; 34(1):90-9.

51 Besarab A, Chernyavskaya E, Motylev I, Shutov E, Kumbar LM, Gurevich K, et al. Roxadustat (FG-4592): correction of anemia in incident dialysis patients. J Am Soc Nephrol. 2016 Apr;27(4):1225-33. 